\title{
Physical activity and general cognitive functioning: A Mendelian Randomization study
}

Boris Cheval ${ }^{1,2^{*}}$, Liza Darrous ${ }^{3,4 \dagger}$, Karmel W. Choi ${ }^{5,6}$, Yann C. Klimentidis ${ }^{7}$, David A. Raichlen $^{8}$, Gene E. Alexander ${ }^{9,10,11,12}$, Stéphane Cullati ${ }^{13}$, Zoltán Kutalik ${ }^{3,4}$, Matthieu P. Boisgontier ${ }^{14,15}$

${ }^{1}$ Swiss Center for Affective Sciences, University of Geneva, Geneva, Switzerland

${ }^{2}$ Laboratory for the Study of Emotion Elicitation and Expression (E3Lab), Department of Psychology, University of Geneva, Geneva, Switzerland

${ }^{3}$ University for Primary Care and Public Health, University of Lausanne, Switzerland

${ }^{4}$ Swiss Institute of Bioinformatics, Lausanne, Switzerland

${ }^{5}$ Department of Epidemiology, Harvard. T.H. Chan School of Public Health, Massachusetts, MA, USA

${ }^{6}$ Department of Psychiatry, Massachusetts General Hospital, Massachusetts, Boston MA, USA

${ }^{7}$ Department of Epidemiology and Biostatistics, University of Arizona, AZ, USA

${ }^{8}$ Human and Evolutionary Biology Section, Department of Biological Sciences, University of Southern California, Los Angeles, CA, USA

${ }^{9}$ Department of Psychology, University of Arizona, AZ, USA

${ }^{10}$ Department of Psychiatry, University of Arizona, AZ, USA

${ }^{11}$ Evelyn F. McKnight Brain Institute, University of Arizona, AZ, USA

${ }^{12}$ Arizona Alzheimer's Disease Consortium, AZ, USA

${ }^{13}$ Population Health Laboratory, Department of Community Health, University of Fribourg, Fribourg, Switzerland

${ }^{14}$ School of Rehabilitation Sciences, Faculty of Health Sciences, University of Ottawa, Ottawa, ON, Canada

${ }^{15}$ Bruyère Research Institute, Ottawa, ON, Canada

*Corresponding author: Campus, Biotech, Chemin des mines 9, 1202 Genève, Switzerland; boris.cheval@unige.ch (B. Cheval)

†Corresponding author for statistical analyses: Route de Berne 113, 1010 Lausanne, Switzerland; liza.darrous@unil.ch (L. Darrous) 


\section{ORCID iDs}

Boris Cheval: 0000-0002-6236-4673

Liza Darrous: 0000-0002-0056-4127

Karmel W. Choi: 0000-0002-3914-2431

Yann C. Klimentidis: 0000-0002-6065-4044

David Raichlen: 0000-0002-4940-7886

Gene E. Alexander: 0000-0002-6476-5606

Stéphane Cullati: 0000-0002-3881-446X

Zoltán Kutalik: 0000-0001-8285-7523

Matthieu P. Boisgontier: 0000-0001-9376-3071

\section{Classification}

Social Sciences; Psychological and Cognitive Sciences

\section{Keywords}

Physical activity; Cognition; Exercise; Mendelian randomization analysis; Accelerometer

\section{Author contributions}

B.C., M.P.B conceived the study. L.D., Z.K. analyzed the data. B.C., M.P.B., L.D, Z.K. drafted the manuscript. All authors critically appraised the manuscript, worked on its content, and approved its submitted version.

\section{Competing interests}

The authors declare no conflict of interests.

\section{Ethical approval}

This study was approved by the Ethics Committee of Geneva Canton, Switzerland (CCER2019-00065).

\section{Data sharing}

The datasets used for the analysis are openly available from the Neale Lab GWAS results at http://www.nealelab.is/uk-biobank and from the Social Science Genetic Association Consortium Downloads at https://www.thessgac.org/data. Only the new GWAS dataset created for the fractions of physical activity are available with permission from the UK Biobank 
https://www.ukbiobank.ac.uk/. The LHC-MR code is available at https:/github.com/LizaDarrous/LHC-MR/.

\section{Acknowledgements}

B.C. is supported by an Ambizione grant (PZ00P1_180040) from the Swiss National Science Foundation (SNSF). Y.C.K. is supported by the National Institute of Health (R01 HL136528).

The authors are thankful to Gail Davies for providing useful comments on the manuscript.

\section{Word count}

Significance: 104

Abstract: 195

Manuscript, characters including spaces (whole document): 4971

Tables: 2

Figures: 2 


\begin{abstract}
Physical activity and cognitive functioning are strongly intertwined. However, the causal relationships underlying this association are still unclear. Physical activity can enhance brain functions, but healthy cognition may also promote engagement in physical activity. Here, we used Latent Heritable Confounder Mendelian Randomization (LHC-MR) to assess the bidirectional relations between physical activity and general cognitive functioning. Association data were drawn from two large-scale genome-wide association studies (UK Biobank and COGENT) on accelerometer-based physical activity $(\mathrm{N}=91,084)$ and cognitive functioning $(\mathrm{N}$ $=257,841)$. We observed a significant MR association, suggesting that increased duration of physical activity improves cognitive functioning $\left(\mathrm{b}=0.61, \mathrm{CI}_{95 \%}=[0.36,0.86], \mathrm{P}=1.16 \mathrm{e}-06\right)$. In contrast, we found no evidence for a causal effect of cognitive functioning on physical activity. Follow-up analyses revealed that the favorable association from physical activity to cognitive functioning was driven by moderate physical activity $\left(b=1.33, \mathrm{CI}_{95 \%}=[0.72,1.94]\right.$, $\mathrm{P}=2.01 \mathrm{e}-05$ ) with no contribution from vigorous physical activity. These findings provide new evidence supporting a beneficial causal effect of moderate physical activity on cognitive functioning. Therefore, interventions that promote moderate rather than vigorous physical activity may be best suited to improve or recover cognitive skills.
\end{abstract}

\title{
Significance Statement
}

Whether the relationship between physical activity and cognitive functioning is a one or twoway association is still unclear. Here, based on a genetically informed method designed to investigate causal relations in observational data, we found a one-way association: Higher levels of physical activity improved cognitive functioning. Results further revealed that only moderate, but not vigorous, physical activity demonstrated a positive effect on cognitive functioning. These findings show that moderate physical activity plays a fundamental role in improving general cognitive functioning, suggesting that policies for healthy ageing and interventions targeting cognitive skills in healthy and clinical populations should primarily rely on this physical activity intensity. 


\section{Introduction}

Promoting healthy ageing is a public health priority $(1,2)$. Physical activity and cognitive functioning are prime targets of this advocacy because their decline is associated with increased disability and mortality (3-7). Multiple cross-sectional and longitudinal studies have shown that physical activity and cognitive functioning are strongly intertwined and decline through the course of life (8-12). However, the causality of this relationship is still unclear. Previous results have indicated that physical activity may improve cognitive functioning (13-19), but recent studies have also suggested that well-functioning cognitive skills can influence engagement in physical activity $(8,20-24)$.

Several mechanisms could explain how physical activity enhances general cognitive functioning (19, 25-31). For example, physical activity can increase brain plasticity, angiogenesis, synaptogenesis, and neurogenesis primarily through the upregulation of growth factors (e.g., brain-derived neurotrophic factor; BDNF) $(27,28,30)$. In addition, the repetitive activation of higher-order brain functions (e.g., planning, inhibition, and reasoning) required to engage in physical activity may contribute to the improvement of these functions $(31,32)$. In turn, other mechanisms could explain how cognitive functioning may affect physical activity. For example, cognitive functioning may be required to counteract the innate attraction to effort minimization and thereby influence a person's ability to engage in physically active behavior (33-36). Of note, these mechanisms are not mutually exclusive and could therefore lead to bidirectionally reinforcing relationships (i.e., positive feedback loop) between physical activity and cognitive functioning (37).

Although previous findings point to a potential mutually beneficial role between physical activity and cognitive functioning, these findings mainly stem from observational designs and analytical methods that cannot fully rule out the influence of social, behavioral, and genetic confounders (37). Accordingly, evidence for a one or two-way association between physical activity and cognitive functioning could be considered weak. Mendelian Randomization (MR) is a statistical approach that can overcome this weakness of observational studies. Specifically, MR uses genetic variants that are randomly distributed in a population as instruments to reduce the risk of confounding or reverse causation $(38,39)$. MR-based effect estimates rely on three main assumptions (40) stating that genetic instruments i) are strongly associated with the exposure (relevance assumption), ii) are independent of confounding factors of the exposureoutcome relationship (independence assumption), and iii) are not associated to the outcome 
conditional on the exposure and potential confounders (exclusion restriction assumption). Wellpowered genome-wide association studies (GWAS) offer multiple genetic instruments that are strongly associated with the exposures of interest (cognitive functioning or physical activity in our case), which validates the relevance assumption. Each such genetic variant (instrument) provides a causal effect estimate of the exposure on the outcome, which can be in turn combined using inverse-variance weighting (IVW) to obtain an overall estimate. The second and third assumptions are less easily validated and can be violated in the case of a heritable confounder affecting the exposure-outcome relationship and biasing the causal estimate. Such confounders can give rise to instruments with proportional effects on the exposure and outcome, hence violating the INstrument Strength Independent of Direct Effect (InSIDE) assumption requiring the independence of the exposure and direct outcome effects.

There have been several extensions to the common IVW method of MR analysis, including MR-Egger, which allows for directional pleiotropy of the instruments and attempts to correct the causal regression estimate. Other extensions, such as median and mode-based estimators, assume that at least half of or the most "frequent" genetic instruments are valid/non-pleiotropic. However, despite the extensions and their assumptions, these methods still suffer from two major limitations. First, they only use a subset of markers as instruments (genome-wide significant markers), which often dilutes the true relationship between traits. Second, they ignore the presence of a latent heritable confounder of the exposure-outcome relationship.

The present study applies the Latent Heritable Confounder MR (LHC-MR) method (41), which addresses the aforementioned limitations, to simultaneously estimate the bidirectional causal effects between physical activity and cognitive functioning, while accounting for possible heritable confounders of their relationship (e.g., body mass index, educational attainment, level of physical activity at work, and material deprivation). Unlike standard MR, LHC-MR accounts for sample overlap from genome-wide genetic instruments, thereby allowing the exposure and the outcome to originate either from overlapping datasets or the same dataset.

Here, the causal estimates were modelled based on summary statistics from large-scale GWAS of accelerometer-based physical activity (42), general cognitive functioning (43, 44), body mass index (45), and educational attainment (45). Finally, it has been suggested that the effects of physical activity on cognitive functioning depend on its intensity, with moderate intensity having a greater beneficial impact compared to vigorous intensity (46-50). Accordingly, we 
assessed whether the causal effect estimates on cognitive functioning were dependent on physical activity intensity (i.e., moderate vs. vigorous).

\section{Results}

As a first proxy for physical activity, two kinds of summary statistics of average acceleration measures were available. To separate moderate from vigorous activity genetics, we conducted GWASs in the UK Biobank with outcomes defined as a fraction of time spent doing moderate (100 to $<425 \mathrm{mg}$ ) and vigorous ( $\geq 425 \mathrm{mg}$ ) physical activity (Manhattan plots and Q-Q plots can be found in Supplementary materials 1 and 2). Thus, the two datasets of average acceleration summary statistics (model 1 and 2), alongside the moderate and vigorous physical activity summary statistics were used in LHC-MR to investigate the possible bidirectional effect that exists between them and cognitive functioning.

\section{Overall average acceleration and general cognitive functioning}

LHC-MR applied to summary statistics belonging to model 1 showed a potential causal effect of average acceleration on cognitive functioning $\left(\mathrm{b}=0.61, \mathrm{CI}_{95 \%}=[0.36,0.86], \mathrm{P}=1.16 \mathrm{e}-06\right)$ (Table 1, Figure 2). In the opposite direction, results did not show significant evidence for a causal effect of cognitive functioning on average acceleration $\left(b=-0.005, \mathrm{CI}_{95 \%}=[-0.06,0.05]\right.$, $\mathrm{P}=0.87)$. Standard MR methods such as IVW, MR Egger, weighted median, simple mode, and weighted mode yielded non-significant causal estimates in either direction (Table 2), using 146 genome-wide significant SNPs as instruments for cognitive functioning and 8 SNPs for average acceleration. LHC-MR applied to summary statistics belonging to model 2 showed consistent results with that of model 1, i.e. a protective effect of accelerometer-based physical activity on cognitive functioning $\left(\mathrm{b}=0.61, \mathrm{CI}_{95 \%}=[0.36,0.86], \mathrm{P}=1.4 \mathrm{e}-06\right)$ and a non-significant causal estimate in the reverse direction $\left(b=0.035, \mathrm{CI}_{95 \%}=[-0.08,0.15], \mathrm{P}=0.56\right)$. Both models showed evidence for the presence of a confounder with a strong negative effect on physical activity (b $\left.=-0.19, \mathrm{CI}_{95 \%}=[-0.26,-0.12], \mathrm{P}=2.65 \mathrm{e}-07\right)$ and a positive impact on cognitive functioning $(\mathrm{b}$ $\left.=0.48, \mathrm{CI}_{95 \%}=[-0.56,-0.4], \mathrm{P}=1.12 \mathrm{e}-34\right)$. Due to the similarity in results between model 1 and 2 , further analyses were conducted using model 1 only.

\section{Moderate physical activity and general cognitive functioning}

LHC-MR applied to the fraction of accelerations corresponding to moderate physical activity showed a potential causal effect of moderate physical activity on cognitive functioning $(b=$ $\left.1.33, \mathrm{CI}_{95 \%}=[0.72,1.94], \mathrm{P}=2.01 \mathrm{e}-05\right)$ (Table 1, Figure 2). This effect was about twice as 
strong in magnitude as that of the positive causal effect of average accelerometer-based physical activity on cognitive functioning. There was also evidence for the presence of a confounder with a strong positive effect on cognitive functioning $\left(b=0.597, \mathrm{CI}_{95 \%}=[0.50,0.69], \mathrm{P}=6.11 \mathrm{e}\right.$ $34)$ and a negative effect on moderate physical activity $\left(\mathrm{b}=-0.236, \mathrm{CI}_{95 \%}=[-0.35,-0.13], \mathrm{P}=\right.$ 2.30e-05). However, it should be noted that a competing model with a very close likelihood shows a large negative, rather than positive, effect of moderate physical activity on cognitive functioning $\left(\mathrm{b}=-1.12, \mathrm{CI}_{95 \%}=[-1.66,-0.58], \mathrm{P}=4.08 \mathrm{e}-05\right)$. Two arguments support the positive, rather than the negative, causal effect. First, a detrimental effect of moderate physical activity on cognitive functioning is highly unlikely, especially in light of the beneficial effect of the average acceleration. Second, the negative (moderate physical activity to cognitive functioning) causal effect model includes a confounder with positive effects on both traits (Supplementary material 3 for the top ten competing maximum likelihood estimates and their parameter estimates), which is in sharp contrast to an opposite effect confounder evidenced by the best fitting model for the average acceleration - cognitive functioning relationship. Accordingly, even if a critical look at the results seems to support a positive causal effect from moderate physical activity to cognitive function, we cannot draw definite conclusions.

\section{Vigorous physical activity and general cognitive functioning}

LHC-MR applied to the fraction of accelerations corresponding to vigorous physical activity on cognitive functioning showed no evidence of a causal effect in either direction (Table 1, Figure 2), with a non-significant causal effect from vigorous physical activity on cognitive functioning $\left(\mathrm{b}=0.07, \mathrm{CI}_{95 \%}=[-0.08,0.21], \mathrm{P}=0.37\right)$, and a similarly non-significant causal effect in the reverse direction $\left(b=0.07, \mathrm{CI}_{95 \%}=[-0.08,0.21], \mathrm{P}=0.36\right)$.

\section{Educational attainment and body mass index as possible confounders}

Since the results of LHC-MR applied to average acceleration and cognitive functioning indicated the presence of a confounder with opposite effect on the two traits, we sought to identify potential confounders. As the results for model 1 and 2 were similar, covariates of model 2 (level of activity at work, extent of walking or standing at work, and Townsend deprivation index) are unlikely confounders. Instead, we investigated the level of educational attainment and body mass index as possible confounders of the two traits. One potential reason for this is that higher education may lead to a less physically active job type, reducing overall physical activity levels, while increasing cognitive functioning. We ran standard MR analyses with educational attainment as exposure and alternated accelerometer-based physical activity 
and cognitive functioning as outcome. We also ran the reverse analyses to obtain reverse causal estimates. Results showed a significant causal effect of educational attainment on cognitive functioning $\left(\mathrm{b}=0.67, \mathrm{CI}_{95 \%}=[0.56,0.78], \mathrm{P}=4.46 \mathrm{e}-34\right)$ using IVW with 33 SNPs as valid instruments. However, educational attainment had a non-significant causal effect on accelerometer-based physical activity, regardless of the MR method used with 34 valid SNPs. Thus, educational attainment is unlikely to be a confounder. The causal effects of body mass index on cognitive functioning $(\mathrm{b}=-0.12, \mathrm{P}=1.01 \mathrm{e}-08)$ and average accelerometer-based physical activity $(b=-0.21, P=4.11 \mathrm{e}-20)$ did not match the opposite signs of the confounder effects found by LHC-MR.

Since the results of LHC-MR applied to moderate acceleration and cognitive functioning indicated the presence of a confounder with opposite effect on the two traits, educational attainment and body mass index were tested as potential confounders. Results showed no significant effect of educational attainment on moderate physical activity, thereby suggesting that educational attainment was not a confounder. Body mass index had a significant effect on moderate physical activity $(b=-0.19, P=4.15 e-17)$ with a similar sign to its effect on cognitive functioning $(\mathrm{b}=-0.12, \mathrm{P}=1.01 \mathrm{e}-08)$, making it a potential confounder of the two traits, which would give rise to causal effects in the same direction. However, this latter causal association is likely to be invalid due to effect size estimates confounded by population stratification (details of this analysis can be found in Supplementary material 4).

\section{Discussion}

This study used a genetically informed method that provides evidence of putative causal relations to investigate the bidirectional associations between accelerometer-based physical activity and general cognitive functioning. Drawing on two large-scale GWAS, we found evidence of a potential causal effect, where higher duration of average physical activity led to increased cognitive functioning. In the opposite direction, we did not observe evidence of a causal effect of cognitive functioning on physical activity. Further analyses revealed that moderate physical activity has a positive effect on cognitive functioning. In contrast, pursuing vigorous physical activity may not have beneficial effects on cognitive functioning. Hence, our study suggests a favorable effect of moderate physical activity on cognitive functioning, but does not indicate that increased cognitive functioning promotes engagement in more physical activity. 
Importantly, our method provides evidence for the existence of a strong latent heritable confounder of the relationship between cognitive functioning and physical activity, which may explain previous contradictory results of standard MR. This confounder was shown to have an opposite effect on cognitive functioning and average physical activity, which could have masked positive causal effects in some previous studies. While we are unsure what this confounder may be, two potential candidates were tested: body mass index and educational attainment. The causal effects of body mass index on cognitive functioning and on average physical activity were not compatible with the effect of the confounder found by LHC-MR. Hence, body mass index cannot be the confounder LHC-MR detected for average physical activity. Regarding educational attainment, although this variable seemed to impact only cognitive functioning in our follow-up MR analysis, its effect on physical activity may have been masked by the fact that people with higher educational attainment have less physically active jobs, thereby reducing the total level of physical activity and impacting only leisure-time physical activity. Future studies should distinguish between work-related physical activity and leisure time-related physical activity to reveal potential effects of educational attainment on these two types of physical activity - i.e., a negative effect of higher educational attainment on work-related physical activity, but a positive effect on leisure-time related physical activity.

Regarding moderate physical activity, results revealed the presence of a confounder that is presumably more complex than originally thought. This confounder may consist of several traits with their own effect (opposite in directions) on the exposure and the outcome. A possible confounder of the cognitive functioning and moderate physical activity relationship could be body mass index, as it had agreeing signs in its effect on each of those traits. Specifically, body mass index had a negative effect both on cognitive functioning and moderate physical activity. The confounder discovered by LHC-MR for those traits pairs also had agreeing signs for the effect of the confounder on cognitive functioning and moderate physical activity. The LHCMR model allows the signs of the confounder effects to flip as long as their direction remain in the same relationship. Hence, body mass index is a possible confounder as well as a potential part of the confounders for the cognitive functioning-average physical activity relationship.

Previous reviews and meta-analyses of observational studies showed a beneficial effect of physical activity on cognitive functioning $(13,16,17,31)$. However, the evidence arising from intervention studies was inconclusive $(18,19,21-23,51)$. It has been argued that these inconsistencies may primarily be attributable to the design-specific tools used to assess physical 
activity (21). Specifically, many observational studies rely on self-reported measures of physical activity, whereas intervention studies often rely on device-based measures of physical activity, or had people exercising under monitored conditions. In other words, evidence of a favorable effect of physical activity on cognitive functioning could have emerged because of the self-reported nature of the measures. Yet, in our observational study, results are based on accelerometer-assessed physical activity, thereby partially ruling out this explanation. Therefore, our findings further support the literature that demonstrated a protective causal role of physical activity on cognitive functioning and extend it by doing so using a device-based measure. Our findings are in line with recent MR-based results showing a protective effect of objectively assessed, but not self-reported, physical activity on the risk of depression (37). Of note, results obtained from LHC-MR differed from those obtained with standard MR methods. At least three key differences in the methods can explain this divergence: i) standard MR uses only genome-wide significant markers, ii) standard MR is biased in case of sample overlap (as is the case in this study) and hence their estimate may be biased towards the observational correlation, and iii) LHC-MR explicitly models proportional pleiotropy unlike standard MR. Accordingly, our results obtained from LHC-MR are bound to be more robust than those obtained from standard MR.

To the best of our knowledge, our study is the first to investigate the potential causal relationship between physical activity and cognitive functioning using such a genetically informed method. We are aware of only two other studies that examined the potential bidirectional associations between physical activity and cognitive functioning $(8,20)$. In contrast to the present study, those two studies observed a positive influence of cognitive functioning on physical activity. At least two factors can explain the differences in the results observed. First, both those studies are based on longitudinal assessment (Granger causality) of the two traits, while our approach is based on a genetically instrumented causal inference technique (LHC-MR). Second, these studies draw on self-reported physical activity rather than device-based measures of physical activity, which may have biased the observed associations between cognitive functioning and physical activity. Self-reported and accelerometer-based physical activity have very modest correlation (52), which reduces the reliability of self-reportbased physical activity studies. Furthermore, self-report bias is correlated with education and cognitive functioning, which questions the use of self-reported physical activity in relationship with cognitive functioning, which itself, is related to the self-report bias. 
Results showed that the beneficial effect of physical activity on cognitive functioning was observed for moderate physical activity, but not for vigorous physical activity. Although these results for moderate physical activity need to be interpreted with caution (see results section), they highlight the potential critical role of physical activity of moderate intensity on cognitive functioning. These results are consistent with previous literature that have suggested that moderate physical activity yields higher benefits of cognitive functioning compared to vigorous physical activity (46-50). However, to the best of our knowledge, this study is the first to assess and compare the causal relationships of moderate and vigorous physical activity with cognitive functioning in such a large-scale dataset and with a genetically informed method. Several mechanisms can explain how physical activity enhances brain functioning. Physical activity promotes neurogenesis, gliogenesis, neuronal excitability, angiogenesis, cortical thickness, and growth factor production $(19,25-31)$. Moreover, the benefits of moderate physical activity could be explained by differences in the quantity of hormones released in the blood. For example, one study observed an inverted $U$ relationships between physical activity intensity and endocannabinoids, with vigorous intensities reducing the concentrations in peripheral endocannabinoids compared with moderate intensities (53). Consequently, vigorous physical activity may be less effective in enhancing cognitive functions than moderate physical activity. Another potential explanation is the stress response associated with vigorous physical activity yielding a large cortisol release that can have a detrimental effect on aspects of cognitive functioning, such as memory $(54,55)$.

Our results contrast with other studies arguing that cognitive functioning is critical to engage in physical activity (33-35). This difference could be explained in at least two ways. Firstly, previous studies examining the positive effect of cognitive functions on physical activity relied on self-reported physical activity, which can bias the observed associations (8, 24, 34). Secondly, our study relied on general cognitive functioning, whereas previous results highlight the specific importance of inhibition resources that may be required to counteract an innate tendency for effort minimization (33-36). Therefore, future studies should investigate the specific relationships between motor inhibition and physical activity.

Among the strengths of the current study are the use of large-scale datasets, the reliance on an objective measure of physical activity, and the application of a robust genetically informed method that can estimate causal effects. However, this study has several features that limit the conclusions that can be drawn. First, the measure of cognitive functioning involves multiple 
tests, which reduced the specificity of the cognitive functioning that was assessed. This feature limits the ability to evaluate the putative causal effects between specific cognitive functioning, such as motor inhibition, and physical activity. Second, MR analysis is designed to elucidate life-long exposure effect on life-long outcome (except in special cases when genetic factors have time-dependent effects), thus it is not suited to explore temporal aspects of these causal relationships. Third, 2-sample MR methods require that SNP effects on the exposure are homogeneous between the two samples. Here, because our two samples differ in age, we rely on the assumption that these genetic effects do not change depending on age. This assumption often turns out to be true, although there are rare exceptions (56). Fourth, LHC-MR can be limited by the low heritability of traits causing bimodal/unreliable estimates. Fifth, regarding the identification of potential confounders, it should be noted that classical MR analysis of the body mass index and cognitive functioning relationship may be biased due to parental/dynasty effects, which can be circumvented by between-sib association analysis (57). Likewise, a weakness of LHC-MR is to assume a single confounder (or several ones with similar effects). For the moderate physical activity - cognitive functioning relationship, two competing causal models were equally probable, with markedly different properties. This may suggest that multiple confounders are present, with some of them having similar effect directions on the traits and others having opposing effect directions. Body mass index may be an example of the former kind of confounders.

Our findings provide preliminary support for a unidirectional relation whereby higher levels of moderate physical activity lead to improved cognitive functioning. These results underline the essential role of moderate physical activity in maintaining or improving general cognitive functioning. Therefore, policies for healthy ageing and interventions that promote moderate rather than vigorous physical activity may be best suited to improve or recover cognitive skills.

\section{Methods}

\section{Data sources and instruments}

This study used de-identified GWAS summary statistics from original studies that were approved by relevant ethics committees. The current study was approved by the Ethics Committee of Geneva Canton, Switzerland (CCER-2019-00065). The available summary-level data were based on 257,841 samples for general cognitive functioning, 91,084 samples for accelerometer-based physical activity, 240,547 samples for educational attainment, and 
359,983 sample for body mass index. Participants age ranged from 40 to 69 years in the UK Biobank, and from 8 to 96 years in the COGENT consortium.

\section{Physical activity}

Device-based measure of physical activity was assessed based on summary statistics from a recent GWAS (42) analyzing accelerometer-based physical activity data from the UK Biobank. In the UK Biobank, about 100,000 participants wore a wrist-worn triaxial accelerometer (Axivity AX3) that was set up to record data for seven days. Individuals with less than 3 days (72 h) of data or not having data in each 1-hour period of the 24-h cycle or for whom device could not be calibrated were excluded. Data for non-wear segments, defined as consecutive stationary episodes $\geq 60 \mathrm{~min}$ where all three axes had a standard deviation $<13 \mathrm{mg}$, were imputed. The details of data collection and processing can be found elsewhere (58). We examined three measures derived from the three to seven days of accelerometer wear: overall acceleration average in milli-gravities $(\mathrm{mg})$, fraction of acceleration $>100 \mathrm{mg}$ and $<425 \mathrm{mg}$ to estimate moderate physical activity (59), and fraction of acceleration $\geq 425 \mathrm{mg}$ to estimate vigorous physical activity (59). The GWAS for overall acceleration average $\left(n_{\max }=91,084\right)$ identified 2 independent genome-wide significant SNPs $(\mathrm{P}<5 \mathrm{e}-09)$, with a SNP-based heritability of $\sim 14 \%$. Furthermore, two GWAS models for overall acceleration average were used: one model where the SNP-accelerometer associations had been adjusted for age, sex, genotyping chip, first ten genomic principal components (PC), center, season (month) of wearing accelerometer (model 1) and another model where an extra adjustment had been done for the baseline self-reported level of physical activity at work, the extent of walking or standing at work, and the Townsend Deprivation Index (model 2).

As for the other two physical activity measures, the fractions of accelerations corresponding to moderate and vigorous physical activity were obtained by running new GWAS on the decomposed acceleration data from UK Biobank using the BGENIE software (60). The phenotype for moderate physical activity was limited to acceleration magnitudes ranging from 100 to $<425 \mathrm{mg}$, whereas vigorous physical activity was limited to accelerations magnitudes ranging from 425 to $2000 \mathrm{mg}$. These acceleration fractions were adjusted for age, sex, and the first $40 \mathrm{PC}$, and the analyzed individuals were restricted to unrelated white-British. The two datasets of average acceleration summary statistics, alongside the moderate and vigorous physical activity summary statistics were used in LHC-MR to investigate the possible bidirectional effect that exists between these summary statistics and cognitive functioning. 


\section{General cognitive functioning}

General cognitive functioning was assessed based on summary statistics from a recent GWAS combining cognitive and genetic data from the UK Biobank and the COGENT consortium $(\mathrm{N}=257,841)(43)$. In the UK Biobank $\left(n_{\max }=222,543\right)$ participants were asked to complete 13 multiple-choice questions that assessed verbal and numerical reasoning. The verbal and numerical reasoning score was based on the number of questions answered correctly within a two-minute time limit. Each respondent took the test up to four times. The phenotype consists of the mean of the standardized score across the measurement occasions for a given participant. In the COGENT consortium $\left(n_{\max }=35,298\right)$, general cognitive function is statistically derived from a principal components analysis of individual scores on a neuropsychological test battery (61). The phenotype estimates overall cognitive functioning and is relatively invariant to the battery used and specific cognitive abilities assessed $(62,63)$. These COGENT data used to assess general cognitive functioning were also used in another GWAS study (44). The GWAS identified 226 independent genome-wide significant SNPs, with a SNP-based heritability of $\sim 20 \%$.

\section{Educational attainment}

Educational attainment was measured in the UK Biobank as the age when completing full-time education $(\mathrm{N}=240,547)(45)$. All cohort-level analyses were restricted to European-ancestry individuals that passed quality control procedures and were at least 30 years of age when their EduYears was reported. The International Standard Classification of Education (ISCED) was used to construct the EduYears phenotype by mapping each major educational qualification with a years-of-education equivalent. The GWAS identified 49 independent genome-wide significant SNPs, with a SNP-based heritability of $\sim 10 \%$.

\section{Body mass index}

Body mass index was derived from the UK Biobank data on height and weight collected at baseline $(\mathrm{N}=359,983)$ (45). Height was measured in whole centimeters with a Seca 2020 stadiometer device. Weight was measured to the nearest $0.1 \mathrm{~kg}$ with the BC-418 MA body composition analyzer (Tanita Corp). Body mass index was derived from weight in $\mathrm{kg}$ divided by height in squared meters. The GWAS identified 615 independent genome-wide significant SNPs, with a SNP-based heritability of $\sim 25 \%$. 


\section{Statistical analysis}

MR is a statistical method in which the randomized inheritance of genetic variation is treated as a natural experiment to estimate the potential causal effect of a modifiable risk factor or exposure on health-related outcomes in an observational design $(38,39)$. MR draws on the assumption that genetic variants, because they are randomly allocated at conception, are less dependent on other risk factors that may be confounders of the exposure and the outcome, and are immune to reverse causality since diseases or health-related outcomes have no reverse effect on genetic variants. Consequently, these genetic variants can be used as instrumental variables, potentially making MR less vulnerable to confounding or reverse causation than conventional approaches in observational studies $(38,39)$. However, MR and its various extensions that aim to relax several of its assumptions are still prone to biased estimates due to the presence of unmeasured genetic confounders (horizontal pleiotropy) and their use of only genome-wide significant markers from GWAS summary statistics.

LHC-MR (41) also uses GWAS summary statistics, but it uses whole genome-wide genetic instruments to estimate bidirectional causal effects, direct heritability, confounder effects while accounting for sample overlap from genome-wide genetic instruments. LHC-MR extends the standard two-sample MR by modeling a latent (unmeasured) heritable confounder that has an effect on the exposure and outcome traits. This allows LHC-MR to differentiate SNPs based on their co-association to a pair of traits and distinguish heritable confounding that leads to genetic correlation from actual causation. Thus, the unbiased bidirectional causal effect between these two traits are estimated simultaneously along with the confounder effect on each trait (Figure 1). LHC-MR is analogous to linkage disequilibrium score regression (LDSC) (64) that estimates trait heritability, in that it uses all genetic markers in a random effect model framework to estimate bidirectional causal effect, as well as other parameters. The LHC-MR framework, with its multiple pathways through which SNPs can have an effect on the traits, as well as its allowance for null effects, make LHC-MR more precise at estimating causal effects compared to standard MR methods.

The likelihood function for LHC-MR derived from the mixture of different pathways that the genome-wide SNPs can have an effect through (acting on either the exposure, the outcome, the confounder, or the combinations of these three), is then optimized given random starting values for the parameters it can estimate. The optimization of the likelihood function then yields the maximum likelihood estimate (MLE) value for a set of estimated parameters, including the 
bidirectional causal effect between the exposure and the outcome as well as the strength of the confounder effect on each of those two traits.

The significance of each of the parameters estimated using LHC-MR was obtained by removing each of the parameters at a time from the likelihood function and then optimizing it again to obtain a second MLE, which would be compared to the first MLE using the likelihood ratio test (LRT) where a P-value is calculated. Furthermore, the causal estimates obtained from LHCMR are on the scale of 1 standard deviation (SD) outcome difference upon a 1 SD exposure change due to the use of standardized summary statistics for the two traits. When LHC-MR revealed a significant confounder effect acting on both exposure and outcome, we attempted to discover what that confounder may be by testing the effect of possible confounders candidates on the exposure trait and outcome trait individually using standard MR. We investigated the roles of education level and body mass index as possible confounders and tried to match their causal effects to those of our estimated confounder effect on the traits. 


\section{References}

1. Kontis V, et al. (2017) Future life expectancy in 35 industrialised countries: projections with a Bayesian model ensemble. Lancet 389:1323-1335.

2. $\quad$ Rechel B, et al. (2013) Ageing in the European union. Lancet 381:1312-1322.

3. Clegg A, Young J, Iliffe S, Rikkert MO, \& Rockwood K (2013) Frailty in elderly people. Lancet 381:752-762.

4. Boyle PA, Buchman AS, Wilson RS, Leurgans SE, \& Bennett DA (2010) Physical frailty is associated with incident mild cognitive impairment in community-based older persons. J Am Geriatr Soc 58:248-255.

5. Lee I-M, et al. (2012) Effect of physical inactivity on major non-communicable diseases worldwide: an analysis of burden of disease and life expectancy. Lancet 380:219-229.

6. $\quad$ Rowe JW \& Kahn RL (1997) Successful aging. Gerontologist 37:433-440.

7. Aichele S, et al. (2018) Fluid intelligence predicts change in depressive symptoms in later life: the Lothian Birth Cohort 1936. Psychol Sci:1-12.

8. Cheval B, et al. (2020) Relationship between decline in cognitive resources and physical activity Health Psychol 39:519-528.

9. Cheval B, et al. (2018) Effect of early-and adult-life socioeconomic circumstances on physical inactivity. Med Sci Sports Exerc 50:476-485.

10. DiPietro L (2001) Physical activity in aging: changes in patterns and their relationship to health and function. $J$ Gerontol A Biol Sci 56:13-22.

11. Levy R (1994) Aging-associated cognitive decline. Int Psychogeriatr 6:63-68.

12. Sebastiani P, et al. (2020) Patterns of multi-domain cognitive aging in participants of the Long Life Family Study. Geroscience.

13. Baumgart $\mathrm{M}$, et al. (2015) Summary of the evidence on modifiable risk factors for cognitive decline and dementia: a population-based perspective. Alzheimer Dement 11:718-726.

14. Blondell SJ, Hammersley-Mather R, \& Veerman JL (2014) Does physical activity prevent cognitive decline and dementia?: A systematic review and meta-analysis of longitudinal studies. BMC Public Health 14:510.

15. Hamer M, Terrera GM, \& Demakakos P (2018) Physical activity and trajectories in cognitive function: English Longitudinal Study of Ageing. J Epidemiol Community Health 72:477-483.

16. Morgan GS, et al. (2012) Physical activity in middle-age and dementia in later life: findings from a prospective cohort of men in Caerphilly, South Wales and a metaanalysis. J Alzheimers Dis 31:569-580.

17. Sofi F, et al. (2011) Physical activity and risk of cognitive decline: a meta-analysis of prospective studies. J Intern Med 269:107-117.

18. Angevaren M, Aufdemkampe G, Verhaar H, Aleman A, \& Vanhees L (2008) Physical activity and enhanced fitness to improve cognitive function in older people without known cognitive impairment. Cochrane Database Syst Rev 3:CD005381.

19. Colcombe S \& Kramer AF (2003) Fitness effects on the cognitive function of older adults: a meta-analytic study. Psychol Sci 14:125-130.

20. Daly M, McMinn D, \& Allan JL (2015) A bidirectional relationship between physical activity and executive function in older adults. Front Hum Neurosci 8:1044.

21. Sabia S, et al. (2017) Physical activity, cognitive decline, and risk of dementia: 28 year follow-up of Whitehall II cohort study. BMJ 357:j2709.

22. Snowden M, et al. (2011) Effect of exercise on cognitive performance in communitydwelling older adults: review of intervention trials and recommendations for public health practice and research. J Am Geriatr Soc 59:704-716. 
bioRxiv preprint doi: https://doi.org/10.1101/2020.10.16.342675; this version posted October 16, 2020. The copyright holder for this preprint (which was not certified by peer review) is the author/funder, who has granted bioRxiv a license to display the preprint in perpetuity. It is made available under aCC-BY 4.0 International license.

23. Young J, Angevaren M, Rusted J, \& Tabet N (2015) Aerobic exercise to improve cognitive function in older people without known cognitive impairment. Cochrane Database Syst Rev 4:CD005381.

24. Lindwall M, et al. (2012) Dynamic associations of change in physical activity and change in cognitive function: Coordinated analyses of four longitudinal studies. J Aging Res 2012:793598.

25. Colzato LS, Kramer AF, \& Bherer L (2018) Editorial special topic: enhancing brain and cognition via physical exercise. J Cogn Enhanc 2:135-136.

26. Roig M, Nordbrandt S, Geertsen SS, \& Nielsen JB (2013) The effects of cardiovascular exercise on human memory: a review with meta-analysis. Neurosci Biobehav Rev 37:1645-1666.

27. Cotman CW \& Berchtold NC (2002) Exercise: a behavioral intervention to enhance brain health and plasticity. Trends Neurosci 25:295-301.

28. Hillman CH, Erickson KI, \& Kramer AF (2008) Be smart, exercise your heart: exercise effects on brain and cognition. Nat Rev Neurosci 9:58-65.

29. Lisanne F, Hsu CL, Best JR, Barha CK, \& Liu-Ambrose T (2018) Increased aerobic fitness is associated with cortical thickness in older adults with mild vascular cognitive impairment. J Cogn Enhanc 2:157-169.

30. Cotman CW, Berchtold NC, \& Christie L-A (2007) Exercise builds brain health: key roles of growth factor cascades and inflammation. Trends Neurosci 30:464-472.

31. Raichlen DA \& Alexander GE (2017) Adaptive capacity: an evolutionary neuroscience model linking exercise, cognition, and brain health. Trends Neurosci 40:408-421.

32. Frith E \& Loprinzi P (2018) Physical activity and individual cognitive function parameters: unique exercise-induced mechanisms. JCBPR 7:92-106.

33. Cheval B, et al. (2018) Behavioral and neural evidence of the rewarding value of exercise behaviors: A systematic review. Sports Med 48:1389-1404.

34. Cheval B, et al. (2019) Cognitive resources moderate the adverse impact of poor neighborhood conditions on physical activity. Prev Med 126:105741.

35. Cheval B, et al. (2018) Avoiding sedentary behaviors requires more cortical resources than avoiding physical activity: an EEG study. Neuropsychologia 119:68-80.

36. Cheval B, et al. (2020) Higher inhibitory control is required to escape the innate attraction to effort minimization. Psychol Sport Exerc 51:101781.

37. Choi KW, et al. (2019) Assessment of bidirectional relationships between physical activity and depression among adults: a 2-sample mendelian randomization study. JAMA Psychiat 76:399-408.

38. Davies NM, Holmes MV, \& Smith GD (2018) Reading Mendelian randomisation studies: a guide, glossary, and checklist for clinicians. BMJ 362:k601.

39. Byrne EM, Yang J, \& Wray NR (2017) Inference in psychiatry via 2-sample mendelian randomization-from association to causal pathway? JAMA Psychiat 74:1191-1192.

40. Lawlor DA, Harbord RM, Sterne JA, Timpson N, \& Davey Smith G (2008) Mendelian randomization: using genes as instruments for making causal inferences in epidemiology. Stat Med 27:1133-1163.

41. Darrous L, Mounier N, \& Kutalik Z (2020) Simultaneous estimation of bi-directional causal effects and heritable confounding from GWAS summary statistics. medRxiv:2020.2001.2027.20018929.

42. Klimentidis YC, et al. (2018) Genome-wide association study of habitual physical activity in over 377,000 UK Biobank participants identifies multiple variants including CADM2 and APOE. Int J Obes 42:1161-1176.

43. Lee JJ, et al. (2018) Gene discovery and polygenic prediction from a 1.1-million-person GWAS of educational attainment. Nat Genet 50:1112. 
44. Davies G, et al. (2018) Study of 300,486 individuals identifies 148 independent genetic loci influencing general cognitive function. Nat Commun 9:1-16.

45. Anonymous (Neale's lab http://www.nealelab.is/uk-biobank/.

46. Szuhany KL, Bugatti M, \& Otto MW (2015) A meta-analytic review of the effects of exercise on brain-derived neurotrophic factor. J Psychiatr Res 60:56-64.

47. Bosch BM, Bringard A, Ferretti G, Schwartz S, \& Iglói K (2017) Effect of cerebral vasomotion during physical exercise on associative memory, a near-infrared spectroscopy study. Neurophotonics 4:041404.

48. Chang Y-K, Labban JD, Gapin JI, \& Etnier JL (2012) The effects of acute exercise on cognitive performance: a meta-analysis. Brain Res 1453:87-101.

49. Suwabe K, et al. (2018) Rapid stimulation of human dentate gyrus function with acute mild exercise. PNAS 115:10487-10492.

50. Bosch BM, et al. (2018) Acute physical exercise improves memory consolidation in humans via BDNF and endocannabinoid signaling. BioRxiv:211227.

51. Erickson KI, et al. (2019) Physical activity, cognition, and brain outcomes: a review of the 2018 physical activity guidelines. Med Sci Sports Exerc 51:1242-1251.

52. Lines RL, et al. (2020) Cross-sectional and longitudinal comparisons of self-reported and device-assessed physical activity and sedentary behaviour. J Sci Med Sport 23:831835.

53. Raichlen DA, Foster AD, Seillier A, Giuffrida A, \& Gerdeman GL (2013) Exerciseinduced endocannabinoid signaling is modulated by intensity. Eur J Applied Physiol 113:869-875.

54. Het S, Ramlow G, \& Wolf O (2005) A meta-analytic review of the effects of acute cortisol administration on human memory. Psychoneuroendocrinology 30:771-784.

55. Hill E, et al. (2008) Exercise and circulating cortisol levels: the intensity threshold effect. J Endocrinol Invest 31:587-591.

56. Winkler TW, et al. (2015) The influence of age and sex on genetic associations with adult body size and shape: a large-scale genome-wide interaction study. PLoS Genet 11:e1005378.

57. Brumpton B, et al. (2020) Avoiding dynastic, assortative mating, and population stratification biases in Mendelian randomization through within-family analyses. Nat Commun 11:3519.

58. Doherty A, et al. (2017) Large scale population assessment of physical activity using wrist worn accelerometers: the UK biobank study. Plos One 12:e0169649.

59. Hildebrand M, Van Hees VT, Hansen BH, \& Ekelund U (2014) Age group comparability of raw accelerometer output from wrist-and hip-worn monitors. Med Sci Sports Exerc 46:1816-1824.

60. Bycroft C, et al. (2018) The UK Biobank resource with deep phenotyping and genomic data. Nature 562:203-209.

61. Trampush JW, et al. (2017) GWAS meta-analysis reveals novel loci and genetic correlates for general cognitive function: a report from the COGENT consortium. $\mathrm{Mol}$ Psychiatr 22:336-345.

62. Johnson W, te Nijenhuis J, \& Bouchard Jr TJ (2008) Still just 1 g: Consistent results from five test batteries. Intelligence 36:81-95.

63. Panizzon MS, et al. (2014) Genetic and environmental influences on general cognitive ability: Is ga valid latent construct? Intelligence 43:65-76.

64. Bulik-Sullivan BK, et al. (2015) LD Score regression distinguishes confounding from polygenicity in genome-wide association studies. Nat Genet 47:291-295. 


\section{Figure and tables}

Table 1. LHC-MR results for the association between accelerometer-based physical activity and general cognitive functioning

\begin{tabular}{|c|c|c|c|c|c|c|}
\hline Parameter & h2X & h2Y & tX & tY & $X \rightarrow Y$ & Y $\rightarrow X$ \\
\hline
\end{tabular}

Average accelerometer-based physical activity

\begin{tabular}{|c|c|c|c|c|c|c|}
\hline Estimate & 0.065 & 0.107 & 0.476 & -0.195 & -0.005 & 0.610 \\
\hline P-value & & & $1.12 \mathrm{e}-34$ & $2.65 \mathrm{e}-07$ & 0.870 & $1.16 \mathrm{e}-06$ \\
\hline
\end{tabular}

Moderate accelerometer-based physical activity (fraction of acceleration $>100 \mathrm{mg}$ and $<425 \mathrm{mg}$ )

\begin{tabular}{|c|c|c|c|c|c|c|}
\hline Estimate & 0.062 & 0.052 & 0.597 & -0.236 & 0.675 & 1.331 \\
\hline P-value & & & $6.11 \mathrm{e}-34$ & $2.30 \mathrm{e}-05$ & 0.234 & $2.01 \mathrm{e}-05$ \\
\hline
\end{tabular}

Vigorous accelerometer-based physical activity (fraction of acceleration $\geq \mathbf{4 2 5} \mathbf{~ m g}$ )

\begin{tabular}{|c|c|c|c|c|c|c|}
\hline Estimate & 0.062 & 0.078 & 0.413 & 0.115 & 0.069 & 0.066 \\
\hline P-value & & & $1.38 \mathrm{e}-28$ & 0.072 & 0.358 & 0.368 \\
\hline
\end{tabular}

Notes. Parameters estimates and their p-values correspond to the LHC-MR optimized model with the maximum likelihood. Heritability on exposure (X; cognitive functioning) and outcome (Y; physical activity) shown as $\mathrm{h} 2 \mathrm{X}$ and $\mathrm{h} 2 \mathrm{Y}$, respectively. The effect of the confounder on $\mathrm{X}$ and $\mathrm{Y}$ shown as $\mathrm{tX}$ and $\mathrm{t} Y$, respectively. Bidirectional associations from $\mathrm{X}$ to $\mathrm{Y}(\mathrm{X} \rightarrow \mathrm{Y})$ and $\mathrm{Y}$ to $\mathrm{X}(\mathrm{Y} \rightarrow \mathrm{X})$ are also reported. 
bioRxiv preprint doi: https://doi.org/10.1101/2020.1016.342675; this version posted October 16, 2020. The copyright holder for this preprint (which was not certified by peer review) is the author/funder, who has granted bioRxiv a license to display the preprint in perpetuity. It is made available under aCC-BY 4.0 International license.

Table 2. Standard MR results for the association between accelerometer-based physical activity and general cognitive functioning

\begin{tabular}{|c|c|c|c|c|c|c|}
\hline Exposure & Outcome & MR method & Valid SNPs & Causal estimate & SE & P-value \\
\hline \multicolumn{7}{|c|}{ Average accelerometer-based physical activity - model 1} \\
\hline \multirow[t]{5}{*}{$\mathrm{CF}$} & \multirow[t]{5}{*}{ PA } & MR Egger & 146 & 0.107 & 0.181 & 0.556 \\
\hline & & Weighted median & 146 & -0.029 & 0.036 & 0.422 \\
\hline & & Inverse variance weighted & 146 & -0.022 & 0.031 & 0.488 \\
\hline & & Simple mode & 146 & -0.058 & 0.107 & 0.584 \\
\hline & & Weighted mode & 146 & -0.058 & 0.110 & 0.593 \\
\hline \multirow[t]{5}{*}{ PA } & \multirow[t]{5}{*}{$\mathrm{CF}$} & MR Egger & 8 & -2.240 & 0.973 & 0.061 \\
\hline & & Weighted median & 8 & -0.038 & 0.059 & 0.513 \\
\hline & & Inverse variance weighted & 8 & -0.112 & 0.095 & 0.238 \\
\hline & & Simple mode & 8 & -0.061 & 0.086 & 0.502 \\
\hline & & Weighted mode & 8 & -0.034 & 0.089 & 0.710 \\
\hline
\end{tabular}

Moderate accelerometer-based physical activity (fraction of acceleration $>100 \mathbf{~ m g}$ and $<425 \mathrm{mg}$ )

\begin{tabular}{|l|c|c|c|c|c|c|}
\hline CF & PA & MR Egger & 131 & -0.031 & 0.181 & 0.864 \\
\cline { 3 - 7 } & & Weighted median & 131 & -0.032 & 0.038 & 0.400 \\
\cline { 3 - 7 } & & Inverse variance weighted & 131 & -0.019 & 0.032 & 0.554 \\
\cline { 3 - 7 } & & Simple mode & 131 & -0.053 & 0.103 & 0.610 \\
\cline { 3 - 7 } & & Weighted mode & 131 & -0.025 & 0.090 & 0.780 \\
\hline \multirow{4}{*}{ PA } & CF & MR Egger & 33 & 0.704 & 0.930 & 0.455 \\
\cline { 3 - 7 } & & Weighted median & 33 & 0.010 & 0.037 & 0.785 \\
\cline { 3 - 7 } & & Inverse variance weighted & 33 & 0.041 & 0.045 & 0.365 \\
\cline { 3 - 7 } & & Simple mode & 33 & 0.003 & 0.078 & 0.964 \\
\cline { 3 - 7 } & & Weighted mode & 33 & 0.011 & 0.068 & 0.876 \\
\hline
\end{tabular}

Vigorous accelerometer-based physical activity (fraction of acceleration $\geq 425 \mathrm{mg}$ )

\begin{tabular}{|l|c|c|c|c|c|c|}
\hline CF & PA & MR Egger & 131 & 0.055 & 0.142 & 0.697 \\
\cline { 3 - 7 } & & Weighted median & 131 & -0.034 & 0.035 & 0.328 \\
\cline { 3 - 7 } & & Inverse variance weighted & 131 & 0.006 & 0.025 & 0.824 \\
\cline { 3 - 7 } & Simple mode & 131 & -0.147 & 0.105 & 0.162 \\
\cline { 3 - 7 } & \multirow{4}{*}{ PA } & Weighted mode & 131 & -0.104 & 0.092 & 0.261 \\
\cline { 3 - 7 } & & MR Egger & 22 & -0.057 & 0.915 & 0.951 \\
\cline { 3 - 7 } & & Weighted median & 22 & 0.023 & 0.039 & 0.551 \\
\cline { 3 - 7 } & & Inverse variance weighted & 22 & 0.005 & 0.043 & 0.911 \\
\cline { 3 - 7 } & & Simple mode & 22 & 0.030 & 0.069 & 0.668 \\
\hline & & Weighted mode & 22 & 0.026 & 0.059 & 0.667 \\
\hline
\end{tabular}

Notes. $\mathrm{CF}=$ general cognitive functioning; $\mathrm{PA}=$ accelerometer-based physical activity. Causal estimates from 5 standard MR methods on alternating exposure and outcome traits. For both moderate and vigorous physical activity as exposure, the cutoff was decreased to $10 \mathrm{e}-5$ because of the low number of genome wide significant SNPs to use as instruments. 
Figure 1. Visual representation of the model in LHC-MR

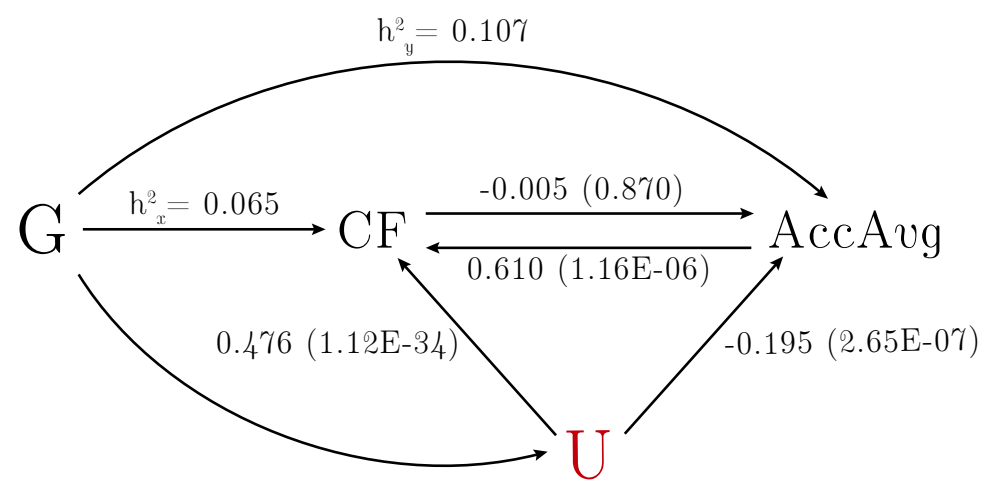

Notes. $\mathrm{G}=$ Genetic instruments; $\mathrm{CF}=$ general cognitive functioning; AccAvg = Average acceleration from device-based measure (model 1); $\mathrm{U}=$ Latent heritable confounder; $\mathrm{h}^{2}{ }_{\mathrm{x}}=$ direct heritability of exposure; $\mathrm{h}^{2} \mathrm{y}=$ direct heritability of the outcome. The figure includes the bidirectional causal effects between the two traits as well as the confounder effects on each of them. Coefficients are beta values. The P-values are indicated in brackets. 
Figure 2. LHC-MR plots for the association between accelerometer-based physical activity and general cognitive functioning

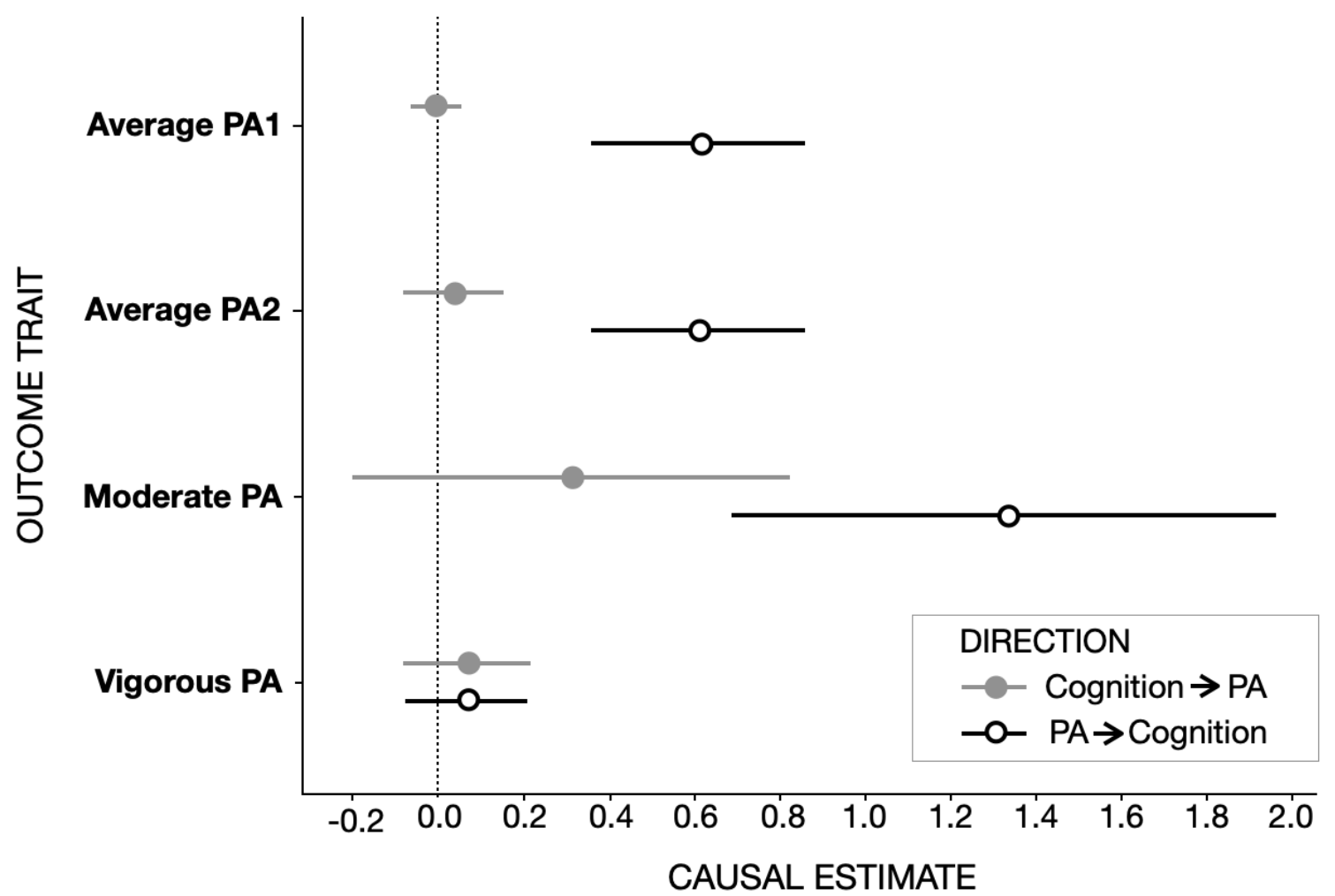

Notes. This modified dot-and-whisker plot reports the causal estimate between general cognitive functioning (cognition) as exposure and varying PA-related traits as outcome. The forward (Cognition $\rightarrow \mathrm{PA})$ and reverse $(\mathrm{PA} \rightarrow$ Cognition) causal estimates are shown in two different colors (grey and black) as dots with 95\% CI whiskers. Average PA2 = average acceleration from device-based measure (model 1). Average PA2 = average acceleration from device-based measure (model 2), Moderate PA = fraction of acceleration corresponding to moderate physical activity. Vigorous $\mathrm{PA}=$ fraction of acceleration corresponding to vigorous physical activity. 